\title{
ABCC subfamily in GtoPdb v.2021.3
}

\author{
Mary Vore ${ }^{1}$
}

1. University of Kentucky, USA

\begin{abstract}
Subfamily ABCC contains thirteen members and nine of these transporters are referred to as the Multidrug Resistance Proteins (MRPs). The MRP proteins are found throughout nature and they mediate many important functions. They are known to be involved in ion transport, toxin secretion, and signal transduction [7, 2].
\end{abstract}

\section{Contents}

This is a citation summary for ABCC subfamily in the Guide to Pharmacology database (GtoPdb). It exists purely as an adjunct to the database to facilitate the recognition of citations to and from the database by citation analyzers. Readers will almost certainly want to visit the relevant sections of the database which are given here under database links.

GtoPdb is an expert-driven guide to pharmacological targets and the substances that act on them. GtoPdb is a reference work which is most usefully represented as an on-line database. As in any publication this work should be appropriately cited, and the papers it cites should also be recognized. This document provides a citation for the relevant parts of the database, and also provides a reference list for the research cited by those parts. For further details see [5].

Please note that the database version for the citations given in GtoPdb are to the most recent preceding version in which the family or its subfamilies and targets were substantially changed. The links below are to the current version. If you need to consult the cited version, rather than the most recent version, please contact the GtoPdb curators.

\section{Database links}

\author{
ABCC subfamily \\ https://www.guidetopharmacology.org/GRAC/FamilyDisplayForward?familyId=153 \\ Transporters \\ MRP1(ABCC1) \\ https://www.guidetopharmacology.org/GRAC/ObjectDisplayForward?objectId=779 \\ MRP2, cMOAT(ABCC2) \\ https://www.guidetopharmacology.org/GRAC/ObjectDisplayForward?objectId=780 \\ MRP3(ABCC3) \\ https://www.guidetopharmacology.org/GRAC/ObjectDisplayForward?objectId=781 \\ MRP4(ABCC4) \\ https://www.guidetopharmacology.org/GRAC/ObjectDisplayForward?objectId=782 \\ MRP5(ABCC5) \\ https://www.guidetopharmacology.org/GRAC/ObjectDisplayForward?objectId=783 \\ MRP6(ABCC6) \\ https://www.guidetopharmacology.org/GRAC/ObjectDisplayForward?objectId=784 \\ SUR1(ATP-binding cassette, sub-family C (CFTR/MRP), member 8) \\ https://www.guidetopharmacology.org/GRAC/ObjectDisplayForward?objectId=2594 \\ SUR2(ABCC9) \\ https://www.guidetopharmacology.org/GRAC/ObjectDisplayForward?objectId=2746 \\ MRP7(ABCC10) \\ https://www.guidetopharmacology.org/GRAC/ObjectDisplayForward?objectId=785 \\ MRP8(ABCC11) \\ https://www.guidetopharmacology.org/GRAC/ObjectDisplayForward?objectId=786
}


MRP9(ABCC12)

https://www.guidetopharmacology.org/GRAC/ObjectDisplayForward?objectId=787

\section{References}

1. Aguilar-Bryan L, Clement JP, Gonzalez G, Kunjilwar K, Babenko A and Bryan J. (1998) Toward understanding the assembly and structure of KATP channels. Physiol Rev 78: 227-45 [PMID:9457174]

2. Alexander SPH, Kelly E, Mathie A, Peters JA, Veale EL, Armstrong JF, Faccenda E, Harding SD, Pawson AJ and Sharman JL et al.. (2019) THE CONCISE GUIDE TO PHARMACOLOGY 2019/20: Transporters. Br J Pharmacol 176 Suppl 1: S397-S493 [PMID:31710713]

3. Bakos E and Homolya L. (2007) Portrait of multifaceted transporter, the multidrug resistanceassociated protein 1 (MRP1/ABCC1). Pflugers Arch 453: 621-41 [PMID:17187268]

4. Borst $P$, de Wolf $C$ and van de Wetering K. (2007) Multidrug resistance-associated proteins 3, 4, and 5. Pflugers Arch 453: 661-73 [PMID:16586096]

5. Buneman P, Christie G, Davies JA, Dimitrellou R, Harding SD, Pawson AJ, Sharman JL and Wu Y. (2020) Why data citation isn't working, and what to do about it Database 2020 [PMID:32367113]

6. Chen ZS, Kawabe T, Ono M, Aoki S, Sumizawa T, Furukawa T, Uchiumi T, Wada M, Kuwano M and Akiyama SI. (1999) Effect of multidrug resistance-reversing agents on transporting activity of human canalicular multispecific organic anion transporter. Mol Pharmacol 56: 1219-28 [PMID:10570049]

7. Dean M, Hamon Y and Chimini G. (2001) The human ATP-binding cassette (ABC) transporter superfamily. J Lipid Res 42: 1007-17 [PMID:11441126]

8. Isomoto S, Kondo C, Yamada M, Matsumoto S, Higashiguchi O, Horio Y, Matsuzawa Y and Kurachi Y. (1996) A novel sulfonylurea receptor forms with BIR (Kir6.2) a smooth muscle type ATP-sensitive K+ channel. J Biol Chem 271: 24321-4 [PMID:8798681]

9. Kalyesubula R and Perazella MA. (2011) Nephrotoxicity of HAART. AIDS Res Treat 2011: 562790 [PMID:21860787]

10. Leier I, Jedlitschky G, Buchholz U, Cole SP, Deeley RG and Keppler D. (1994) The MRP gene encodes an ATP-dependent export pump for leukotriene $\mathrm{C} 4$ and structurally related conjugates. J Biol Chem 269: 27807-10 [PMID:7961706]

11. Priebe W, Krawczyk M, Kuo MT, Yamane Y, Savaraj N and Ishikawa T. (1998) Doxorubicin- and daunorubicin-glutathione conjugates, but not unconjugated drugs, competitively inhibit leukotriene C4 transport mediated by MRP/GS-X pump. Biochem Biophys Res Commun 247: 859-63 [PMID:9647783]

12. Ramaen O, Leulliot N, Sizun C, Ulryck N, Pamlard O, Lallemand JY, Tilbeurgh Hv and Jacquet E. (2006) Structure of the human multidrug resistance protein 1 nucleotide binding domain 1 bound to Mg2+/ATP reveals a non-productive catalytic site. J Mol Biol 359: 940-9 [PMID:16697012]

13. Rehmann H. (2012) Epac2: a sulfonylurea receptor? Biochem Soc Trans 40: 6-10 [PMID:22260657]

14. Reid G, Wielinga P, Zelcer N, van der Heijden I, Kuil A, de Haas M, Wijnholds J and Borst P. (2003) The human multidrug resistance protein MRP4 functions as a prostaglandin efflux transporter and is inhibited by nonsteroidal antiinflammatory drugs. Proc Natl Acad Sci USA 100: 9244-9 [PMID:12835412]

15. Sager G, Ørvoll EØ, Lysaa RA, Kufareva I, Abagyan R and Ravna AW. (2012) Novel cGMP efflux inhibitors identified by virtual ligand screening (VLS) and confirmed by experimental studies. $J$ Med Chem 55: 3049-57 [PMID:22380603]

16. Silbermann K, Stefan SM, Elshawadfy R, Namasivayam V and Wiese M. (2019) Identification of Thienopyrimidine Scaffold as an Inhibitor of the ABC Transport Protein ABCC1 (MRP1) and Related Transporters Using a Combined Virtual Screening Approach. J Med Chem 62: 43834400 [PMID:30925062]

17. Suzuki H and Sugiyama Y. (1998) Excretion of GSSG and glutathione conjugates mediated by MRP1 and cMOAT/MRP2. Semin Liver Dis 18: 359-76 [PMID:9875554]

18. Wängler B, Beck C, Shiue CY, Schneider S, Schwanstecher C, Schwanstecher M, Feilen PJ, Alavi A, Rösch F and Schirrmacher R. (2004) Synthesis and in vitro evaluation of (S)-2([11C]methoxy)-4-[3-methyl-1-(2-piperidine-1-yl-phenyl)-butyl-carbamoyl]-benzoic acid ([11C]methoxy-repaglinide): a potential beta-cell imaging agent. Bioorg Med Chem Lett 14: 5205-9 [PMID:15380228]

19. Zelcer N, Reid G, Wielinga P, Kuil A, van der Heijden I, Schuetz JD and Borst P. (2003) Steroid and bile acid conjugates are substrates of human multidrug-resistance protein (MRP) 4 (ATPbinding cassette C4). Biochem J 371: 361-7 [PMID:12523936]

20. Zhang X, Qiu Y, Li X, Bhattacharjee S, Woods M, Kraft P, Lundeen SG and Sui Z. (2009) Discovery and structure-activity relationships of a novel series of benzopyran-based K(ATP) 
openers for urge urinary incontinence. Bioorg Med Chem 17: 855-66 [PMID:19101153] 